\title{
Como aplicar la innovación docente al enseñar Hockey \\ durante la Covid-19
}

\section{How to apply teaching innovation when teaching Hockey during Covid-19}

Marcos PRAdAs García

https://orcid.org/0000-0002-5104-0681

Universidad de Sevilla

Motricidad humana y Rendimiento Deportivo

mpradas@us.es

DOI: http://dx.doi.org/10.12795/9788447231003.054

Pp.: 1133-1152 


\section{Contexto}

Este ciclo de mejora consta de dos fases CIMA 1 y CIMA 2 , se desarrolla en las clases de la asignatura de Fundamentos de Rugby, Hockey y su enseñanza, en el bloque de Hockey, que se imparte como optativa en tercero del Grado en Ciencias de la Actividad Física de la Facultad de Ciencias de la Educación de la Universidad de Sevilla.

La COVID-19 ha cambiado nuestra forma de relacionarnos. Las clases prácticas de actividad física y deporte no son una excepción. Han surgido medidas como distancia de seguridad, concurrencia, espacios, material, etc. (Gómez Calvo, 2020). Hay que seguir adelante y adaptarse a los nuevos tiempos en la docencia de la actividad física. (Gallardo, 2020).

En la Web del Ministerio de Sanidad, se encuentran nombradas las recomendaciones principales de labores de limpieza, higiene y desinfección de las instalaciones deportivas (Ministerio de Sanidad, 2020c).

Es una asignatura del primer cuatrimestre con 52 alumnos matriculados. Debido a las restricciones por la covid-19 las clases son presenciales para el 33\% de los estudiantes y se retransmiten a través de Blackboard Collaborate ULtra en la plataforma virtual de la Universidad de Sevilla. La clase teórica paso a ser online tras las restricciones marcadas por la Junta de Andalucía el 30/10/20.

Cada subgrupo de clase tiene 4 horas a la semana de clase, repartidas en sesiones de 2 hora de teoría los martes y 2 horas de práctica los lunes, jueves y viernes para cada uno de los tres subgrupos de práctica de la asignatura, dos de turno de mañana (14-16 horas) y uno de turno de tarde (15-17 horas).

Ciclos de Mejora en el Aula (2020). Experiencias de Innovación Docente de la US Esta obra se distribuye con la licencia Creative Commons 


\title{
Diseño previo del CIMA
}

\section{Mapa de contenidos}

\author{
FIGURAS FIDOP \\ Como aplicar la innovación docente al enseñar Hockey \\ durante la Covid-19
}

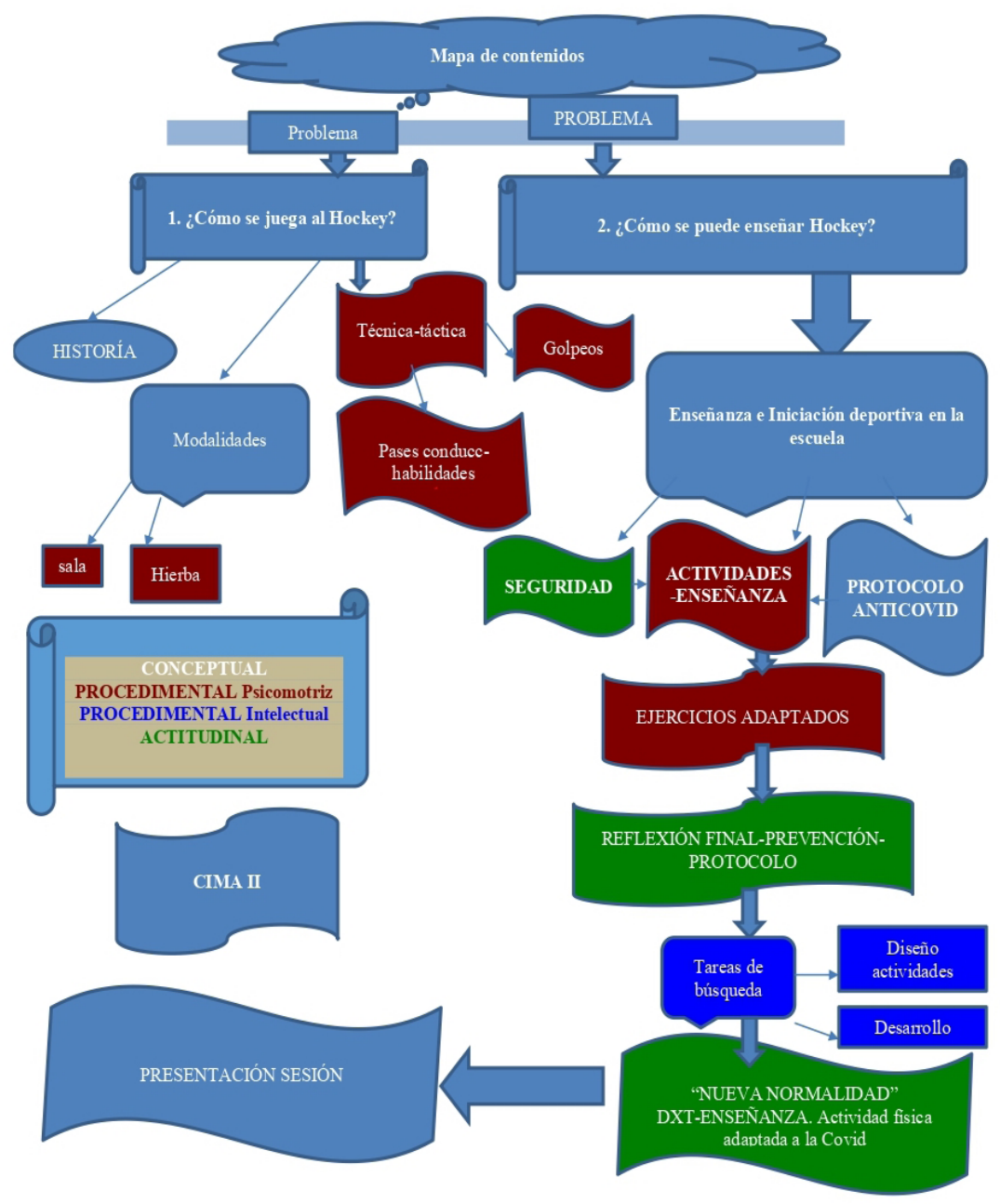

Figura 1. Mapa de contenidos

Ciclos de Mejora en el Aula (2020). Experiencias de Innovación Docente de la US (c) (i) $(-)$ Esta obra se distribuye con la licencia Creative Commons Reconocimiento-NoComercial-SinObraDerivada $\quad 4.0$ Internacional (CC BY-NC-ND 4.0.) 


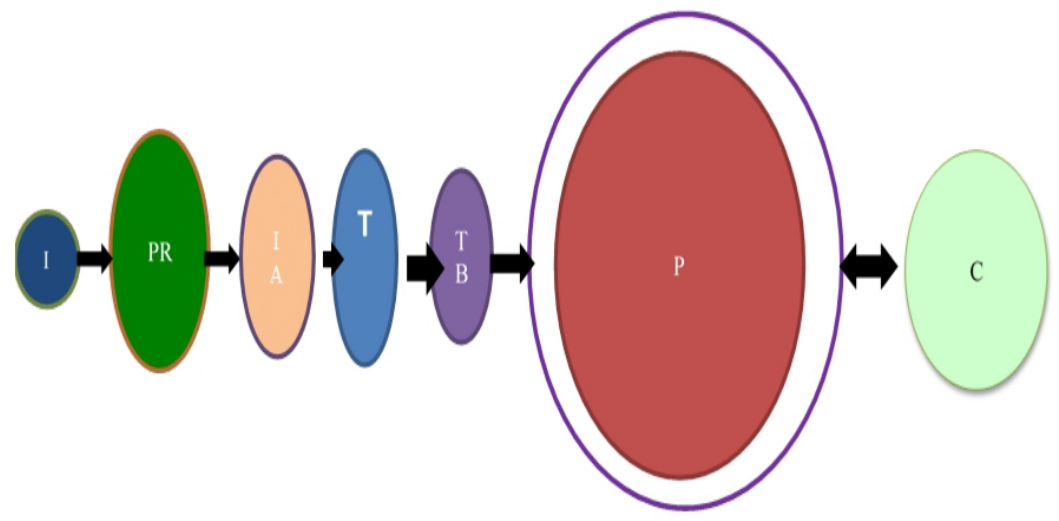

I: Introducción; Pr: Preguntas; IA: Ideas Alumnos; T: Teoria; TB: Tareas de búsqueda; P: Práctica ; $\mathrm{C}$ : Conclusiones

Figura 2. Modelo metodológico posible y secuencia de actividades

El diseño del CIMA 1, se realiza durante 4 horas de clase: 2 de teoría y 2 de práctica. Se sigue un modelo didáctico alternativo donde el alumno es el principal protagonista. Se usa el descubrimiento guiado, la resolución de problemas y el aprendizaje a través de retos. Los alumnos deben diseñar 3 actividades para la práctica de la asignatura que cumplan los protocolos de seguridad antiCovid y sigan los contenidos de fundamentos de enseñanza del deporte: Hockey.

Se inicia la actividad con un debate sobre un concepto, para promover la intervención en la actividad de los alumnos. A continuación, deben realizar una tarea de búsqueda por parejas en bases de datos y la literatura publicada.

Para desarrollar el CIMA 2, se mantiene una estructura similar a la iniciada en el CIMA 1, pero ampliada, trabajando a partir de preguntas y buscando que los alumnos lleven a la práctica lo trabajado en la teoría siguiendo los modelos vistos en Bain (2006), Finkel (2008) y Porlán (2017).

Ciclos de Mejora en el Aula (2020). Experiencias de Innovación Docente de la US Esta obra se distribuye con la licencia Creative Commons 
Se les plantean dos problemas a los alumnos, para que estos los resuelvan y realicen una presentación en la parte práctica de una sesión de trabajo donde se dé respuesta a uno de estos dos problemas. Antes de la teoría, los alumnos expresan sus ideas a través de debates, propuestas o resolución de preguntas relacionadas con las dos principales preguntas de la actividad, se trata de recabar las ideas del alumnado (IA), conocer su opinión antes que realicen las tareas de búsqueda sobre la literatura existente del tema a trabajar.

Se parte de una introducción donde se presenta el tema de la sesión y se busca la interacción del alumnado, realizando una comparativa con sus propias ideas que formularon antes de la teoría. Al final de la sesión se plantea a los alumnos una tarea de búsqueda relacionada con lo que se va a ver en la práctica.

Tras formular las preguntas que supone la continuación del caso práctico y desarrollo de la presentación en cada sesión, se pretende recabar las ideas del alumnado, mediante debate, preguntas y tareas de búsqueda por parejas al final de la clase de teoría (en esta fase se llevará a cabo a partir de la búsqueda de información, el análisis de los datos, la elaboración de argumentos, la puesta en común...), haciendo uso del cuestionario al inicio y final del CIMA 2.

Por último, y tras las presentaciones de todos los grupos de alumnos, se deberá extraer una conclusión de este bloque de trabajo. Esta conclusión se enunciará como cierre de la sesión. En el CIMA 2, este apartado se desarrollará con posterioridad a la clase, al final de la última clase práctica y en la siguiente sesión de teoría con un debate con alumnos y pasando de nuevo el cuestionario.

Ciclos de Mejora en el Aula (2020). Experiencias de Innovación Docente de la US Esta obra se distribuye con la licencia Creative Commons 


\section{Secuencia de actividades}

Se continua lo desarrollado en el CIMA1 en 4 horas y se amplía el trabajo a 8 horas, no solo en la metodología, sino teniendo en cuenta los contenidos, buscando su modificación y centrándonos en lo más interesante del programa y en los que queremos que aprendan nuestros alumnos, usando las actividades para conseguir este objetivo.

Se continua con el trabajo desarrollado en el CIMA 1 donde el alumno tiene que trabajar en grupo e implicarse en obtener su aprendizaje a través de su trabajo y participación en la asignatura.

Los alumnos pasan de tener que diseñar un ejercicio a hacerlo con una sesión y deben partir de dar respuesta a las dos preguntas principales formuladas. En las siguientes tablas se desarrolla la secuencia de actividades.

Ciclos de Mejora en el Aula (2020). Experiencias de Innovación Docente de la US Esta obra se distribuye con la licencia Creative Commons 
Tabla 1. Secuencia de actividades de la sesión primera del problema 1

\begin{tabular}{|c|c|c|c|}
\hline \multicolumn{4}{|c|}{ SESIÓN 1} \\
\hline \multicolumn{4}{|c|}{ PROBLEMA 1: ¿CÓMO SE JUEGA AL HOCKEY? } \\
\hline 1 & INTRODUCCIÓN & $30 \mathrm{~min}$ & \\
\hline \multicolumn{4}{|c|}{$\begin{array}{l}\text { Descripción de la actividad e introducción de la sesión } \\
\text { Presentación del profesor, de la asignatura y explicación de cómo se van a } \\
\text { desarrollar las próximas clases correspondientes a la aplicación del ciclo de } \\
\text { mejora en el aula. } \\
\text { Para aumentar la motivación y participación de los alumnos durante las } \\
\text { próximas clases se les informará de que aquellos que asistan y participen } \\
\text { en todas las actividades del tema obtendrán una puntuación extra. } \\
\text { Se les informará a los alumnos que se va a realizar un cuestionario para } \\
\text { conocer las ideas que tienen sobre el tema que vamos a tratar en los } \\
\text { próximos días. Se les insistirá en que no es un examen y que es anónimo. } \\
\text { Instrucciones: Contesta a las siguientes preguntas del cuestionario } \\
\text { individualmente con las ideas/conocimientos que tengas actualmente, por } \\
\text { favor, no busques en Google ni en apuntes de otros años, me interesa mucho } \\
\text { saber cuál es la información que tienes de partida sobre las cuestiones que } \\
\text { vamos a trabajar en los próximos días. Puedes expresarte con total libertad } \\
\text { ya que este sondeo es anónimo, emplea un nombre inventado, un alias o } \\
\text { un código. } \\
\text { Recurso: Se empleará cuestionario Google doc para favorecer el anonimato } \\
\text { de los estudiantes. Se explica cómo se va a desarrollar la sesión, se } \\
\text { introducen conceptos de teoría de la sesión y se plantean preguntas a los } \\
\text { alumnos. }\end{array}$} \\
\hline 2 & ANTEAMIENTO DEL PROBLEMA & $25 \mathrm{~min}$ & \\
\hline \multicolumn{4}{|c|}{$\begin{array}{l}\text { Descripción de la actividad } \\
\text { Se muestra la pregunta y se relaciona con los conceptos de técnica y táctica } \\
\text { en el hockey y se plantean preguntas a los alumnos sobre como solucionar } \\
\text { este problema }\end{array}$} \\
\hline & IDEAS ALUMNOS & $15 \mathrm{~min}$ & \\
\hline \multicolumn{4}{|c|}{$\begin{array}{l}\text { Los alumnos expresan sus ideas sobre las preguntas formuladas y una vez } \\
\text { que se les ha presentado el problema. Deben de dar soluciones a como } \\
\text { se juega al hockey desde su punto de vista como alumnos de iniciación, } \\
\text { procurando buscar en su experiencia motriz y en relación con otros deportes } \\
\text { vistos a lo largo de lo visto en su etapa universitaria y su vida deportiva. }\end{array}$} \\
\hline & PUESIA EN COMUIN & $20 \mathrm{mi}$ & \\
\hline
\end{tabular}

Ciclos de Mejora en el Aula (2020). Experiencias de Innovación Docente de la US Esta obra se distribuye con la licencia Creative Commons Reconocimiento-NoComercial-SinObraDerivada Internacional (CC BY-NC-ND 4.0.) 
Descripción de la actividad

Se desarrolla la teoría de la sesión, esta documentación los alumnos la tienen colgada en la plataforma, a no ser que el docente considere interesante no colgarla para que los alumnos no tengan información y sigan la explicación del docente a través del PPT, Prezi, etc. Se intercalan preguntas a los alumnos que deben contestar en el momento para afianzar el conocimiento y resolver dudas.

\begin{tabular}{|l|l|l|r|}
\hline 5 & TRABAJO EN GRUPO & $30 \mathrm{~min}$ & TB \\
\hline
\end{tabular}

Descripción de la actividad

Se explicará que se van a formar grupos de 2-3 alumnos. Los estudiantes podrán elegir los compañeros del grupo y deben elegir un portavoz que subirá el trabajo del grupo a la plataforma de la asignatura. Para el trabajo en clase, si esta es online se realizará la creación de los grupos de trabajo en Blackboard Collaborate Ultra. Al final los alumnos pondrán en común las respuestas.

Se desarrollan unas tareas de búsqueda sobre un tema planteado por el profesor relacionado con la pregunta principal y con la aplicación en la práctica.

Tabla 2. Secuencia de actividades de la sesión segunda del problema 1

\begin{tabular}{|c|c|c|c|}
\hline \multicolumn{4}{|c|}{ SESIÓN 2} \\
\hline \multicolumn{4}{|c|}{ PROBLEMA 1: ¿CÓMO SE JUEGA AL HOCKEY? } \\
\hline 1 & INTRODUCCIÓN-PROBLEMA & $20 \mathrm{~min}$ & PR \\
\hline \multicolumn{4}{|c|}{$\begin{array}{l}\text { Descripción de la actividad } \\
\text { Se desarrolla el calentamiento, la organización de la sesión y los materiales } \\
\text { prestando especial atención a las medidas de prevención y protocolo } \\
\text { anticovid y se estructura la sesión por orden, con lugar de exposición de } \\
\text { cada grupo de } 3 \text { alumnos. Se recuerda el problema sobre el cual se basa la } \\
\text { sesión y las medidas de seguridad }\end{array}$} \\
\hline 2 & PRÁCTICA & $80 \mathrm{~min}$ & \\
\hline \multicolumn{4}{|c|}{$\begin{array}{l}\text { Descripción de la actividad } \\
\text { Desarrollo de las presentaciones de los alumnos con } 20 \text { minutos para cada } \\
\text { grupo para exponer su sesión. Cada grupo tiene que realizar entre } 2 \text { y } 3 \\
\text { ejercicios interrelacionados entre si, que cumplan los objetivos de la sesión } \\
\text { y la pregunta a responder, deben ajustar sus ejercicios al tiempo máximo } \\
\text { concedido y ellos son los que dirigen la clase, siendo el docente un mero } \\
\text { observador que no interviene en el desarrollo de la presentación a no ser } \\
\text { que sea imprescindible por algún problema o motivo de seguridad. } \\
\text { El alumno debe dejar constancia de las presentaciones de los compañeros } \\
\text { en el dossier de práctica }\end{array}$} \\
\hline
\end{tabular}

Ciclos de Mejora en el Aula (2020). Experiencias de Innovación Docente de la US Esta obra se distribuye con la licencia Creative Commons Reconocimiento-NoComercial-SinObraDerivada $\quad 4.0$ Internacional (CC BY-NC-ND 4.0.) 


\begin{tabular}{|c|c|c|c|}
\hline & PUESTA EN COMUN & $20 \mathrm{~min}$ & \\
\hline \multicolumn{4}{|c|}{$\begin{array}{l}\text { Descripción de la actividad } \\
\text { Se realiza una reflexión final entre todos los participantes de la sesión. Se } \\
\text { trata de llegar a unas conclusiones finales. Se intentará generar debate y } \\
\text { reflexión entre los estudiantes y consensuar la respuesta final. Es importante } \\
\text { fomentar la participación de los estudiantes y motivarles para den su } \\
\text { opinión de manera sincera. Al final se resumirá el contenido abordado y se } \\
\text { resolverán las dudas generadas }\end{array}$} \\
\hline
\end{tabular}

Tabla 3. Secuencia de actividades de la sesión primera del problema 2

\begin{tabular}{|c|c|c|c|}
\hline \multicolumn{4}{|c|}{ SESIÓN 1} \\
\hline \multicolumn{4}{|c|}{ PROBLEMA 2: ¿CÓMO SE PUEDE ENSEÑAR HOCKEY? } \\
\hline 1 & INTRODUCCIÓN & $30 \mathrm{~min}$ & \\
\hline \multicolumn{4}{|c|}{$\begin{array}{l}\text { Descripción de la actividad e introducción de la sesión } \\
\text { Explicación de cómo se va a desarrollar la sesión. Se introducen conceptos } \\
\text { de teoría de la sesión y se plantean preguntas a los alumnos. Se genera un } \\
\text { debate sobre la importancia de la actividad física en la sociedad y el papel } \\
\text { de la actividad física y el deporte en la escuela y en la enseñanza. }\end{array}$} \\
\hline 2 & PLANTEAMIENTO DEL PROBLEMA & $25 \mathrm{~min}$ & PR \\
\hline \multicolumn{4}{|c|}{$\begin{array}{l}\text { Descripción de la actividad } \\
\text { Se muestra la pregunta y se relaciona con los conceptos de Enseñanza de } \\
\text { Hockey en la escuela y como se introduce este deporte en la enseñanza } \\
\text { secundaría y se plantean preguntas a los alumnos sobre como solucionar } \\
\text { este problema. }\end{array}$} \\
\hline 3 & IDEAS ALUMNOS & $15 \min$ & PR \\
\hline \multicolumn{4}{|c|}{$\begin{array}{l}\text { Los alumnos expresan sus ideas sobre las preguntas formuladas y una vez } \\
\text { que se les ha presentado el problema. Deben de dar soluciones a como } \\
\text { se juega al hockey desde su punto de vista como alumnos de iniciación, } \\
\text { procurando buscar en su experiencia motriz y en relación con otros deportes } \\
\text { vistos a lo largo de la carrera y su vida deportiva. }\end{array}$} \\
\hline 4 & PUESTA EN COMUN & $20 \mathrm{~min}$ & $\mathrm{~T}$ \\
\hline \multicolumn{4}{|c|}{$\begin{array}{l}\text { Descripción de la actividad } \\
\text { Se desarrolla la teoría de la sesión, esta documentación los alumnos } \\
\text { la tienen colgada en la plataforma, a no ser que el docente considere } \\
\text { interesante no colgarla para que los alumnos no tengan información y } \\
\text { siguen la explicación del docente a través del PPT, Prezi, etc. Se intercala con } \\
\text { preguntas a los alumnos que deben contestar en el momento para afianzar } \\
\text { el conocimiento y resolver dudas. }\end{array}$} \\
\hline
\end{tabular}

Ciclos de Mejora en el Aula (2020). Experiencias de Innovación Docente de la US Esta obra se distribuye con la licencia Creative Commons Reconocimiento-NoComercial-SinObraDerivada Internacional (CC BY-NC-ND 4.0.) 
Descripción de la actividad

Se explicará que se van a formar grupos de 2-3 alumnos. Los estudiantes podrán elegir los compañeros del grupo y deben elegir un portavoz que subirá el trabajo del grupo a la plataforma de la asignatura. Para el trabajo en clase, si esta es online se realizará la creación de los grupos de trabajo en Blackboard Collaborate Ultra. Y al final los alumnos pondrán en común las respuestas.

Se desarrollan unas tareas de búsqueda sobre un tema planteado por el profesor relacionado con la pregunta principal y con la aplicación en la práctica.

Tabla 4. Secuencia de actividades de la sesión segunda del problema 2

\section{SESIÓN 2}

PROBLEMA 2: ¿CÓMO SE PUEDE ENSEÑAR HOCKEY?

\begin{tabular}{|l|l|l|l|}
\hline 1 & INTRODUCCIÓN-PROBLEMA & $20 \mathrm{~min}$ & PR \\
\hline
\end{tabular}

Descripción de la actividad

Se desarrolla el calentamiento, la organización de la sesión y los materiales y se estructura la sesión por orden, con lugar de exposición de cada grupo de 3 alumnos. Se recuerda el problema sobre el cual se basa la sesión y las medidas de seguridad

\begin{tabular}{|l|l|l|l|}
\hline 2 & PRÁCTICA & $80 \mathrm{~min}$ & $\mathrm{P}$ \\
\hline
\end{tabular}

Descripción de la actividad

Desarrollo de las presentaciones de los alumnos con 20 minutos para cada grupo para exponer su sesión, cada grupo tiene que realizar entre 2 y 3 ejercicios interrelacionados entre si y que cumplan los objetivos de la sesión y la pregunta a responder, deben ajustar sus ejercicios al tiempo máximo concedido y ellos son los que dirigen la clase, siendo el docente un mero observador que no interviene en el desarrollo de la presentación a no ser que sea imprescindible por algún problema o motivo de seguridad El alumno debe dejar constancia de las presentaciones de los compañeros en el dossier de práctica.

\begin{tabular}{l|l|l|r|}
\hline 3 & \multicolumn{1}{|c|}{ PUESTA EN COMUN } & 20 min & C \\
\hline Descripción de la actividad \\
Se realiza una reflexión final entre todos los participantes de la sesión. Se \\
trata de llegar a unas conclusiones finales. Se intentará generar debate y \\
reflexión entre los estudiantes y consensuar la respuesta final. Es importante \\
fomentar la participación de los estudiantes y motivarles para den su \\
opinión de manera sincera. Al final se resumirá el contenido abordado y se \\
resolverán las dudas generadas
\end{tabular}

Ciclos de Mejora en el Aula (2020). Experiencias de Innovación Docente de la US Esta obra se distribuye con la licencia Creative Commons 


\section{Cuestionario inicial y final}

Se realiza un cuestionario a los alumnos antes y después de realizar el CIMA 2 para analizar los modelos mentales de los estudiantes, queremos conocer su punto de vista sobre el fenómeno estudiado y realizar un análisis sistemático de sus opiniones.

Para la realización del cuestionario se les plantea un caso práctico que se detalla a continuación.

Introducción: Somos el personal de una escuela de Hockey y estamos preparando la programación del año, nos reunimos con los docentes de EF de un centro de secundaría donde queremos realizar una presentación de la escuela. Lleva preparadas tus respuestas al cuestionario para la próxima reunión, pues tenéis que hacer el informe de la reunión y ser capaces de vender vuestra actividad. Quiero que te expreses con el máximo de libertad y con tus puntos de vista, asi que este sondeo es anónimo y lo debatiremos en la reunión sin saber quién lo ha escrito. Argumenta muy bien todas tus opiniones y pon una clave/ Nick para que puedas recuperar tu cuestionario al final de la reunión. Gracias. Coordinadora del Equipo de Seguridad. Gerente Escuela de Hockey. Se pasa a los alumnos por primera vez el 10/11/2020.

1. Define que es para ti Hockey. Justifica tu respuesta

2. Define Seguridad en la actividad física según tu criterio.

3. ¿Cómo se juega al hockey con niñ@s?

4. ¿Cómo se debe enseñar Hockey en nuestra escuela?

5. ¿Qué son para ti las técnicas de prevención y protocolo anticovid?

6. Donde te has documentado para aplicar medidas de prevención en la actividad de Hockey

Ciclos de Mejora en el Aula (2020). Experiencias de Innovación Docente de la US Esta obra se distribuye con la licencia Creative Commons 
7. Qué podemos ofrecer al IES para vender nuestra actividad como extraescolar.

8. Qué criterios sigues para diseñar las actividades

9. Que factores consideras imprescindibles para jugar Hockey en la escuela

10. Qué consideras más importante en la enseñanza del Hockey en la situación actual

\section{Aplicación del CIMA}

\section{Sesión 1}

La sesión ha tenido un importante incremento en la participación por parte del alumnado tanto en el debate y posterior lluvia de ideas para ofrecer soluciones a la pregunta planteada, como a la hora de intervenir, los alumnos, que han resuelto las preguntas formuladas por el docente además de formular preguntas propias relacionadas con la pregunta principal.

La principal fortaleza es el aumento de la participación del alumnado y la debilidad es en este caso la no presencialidad de la clase, que hace más difícil el feedback y la mayor participación de todos los alumnos.

Las mejoras a realizar son proponer temas y contenidos que fomenten el interés y la participación del alumnado, esto se podría conseguir con cuestionarios iniciales y dejar a los alumnos que propongan ideas propias sobre propuestas de mejora y formas de conseguir los objetivos buscados en la asignatura.

Ciclos de Mejora en el Aula (2020). Experiencias de Innovación Docente de la US Esta obra se distribuye con la licencia Creative Commons 


\section{Sesión 2}

La sesión consta de tres presentaciones de los alumnos, la sesión es muy útil y con una valoración muy positiva por cómo se implican los alumnos en crear una sesión original y que resulte amena a sus compañeros y su principal fortaleza es la oportunidad que tienen lo alumnos de mostrar lo aprendido y si han trabajado el diseño y desarrollo de la sesión, llevando ellos mismos la dirección de la clase durante el tiempo asignado.

La debilidad principal son los problemas que tienen los alumnos para automatizar durante la práctica las medidas de seguridad y la no correcta aplicación al 100\% de un protocolo anticovid, siendo esta la principal propuesta de mejora, el saber diseñar y crear actividades y ejercicios que cumplan este protocolo.

\section{Sesión 3}

La sesión ha sido muy positiva por la implicación de los alumnos y la necesidad de resolver por si mismos problemas que genera la situación actual y que se van a encontrar en un futuro en su práctica profesional. Esta sería la principal fortaleza, la aplicación práctica de la sesión y la implicación de los alumnos.

La debilidad ha sido el menor porcentaje de alumnos en la sesión y menor participación, por problemas de acceso en la plataforma a lo largo de la mañana.

Las mejoras para un futuro seria que el docente dispusiera de tiempo suficiente para grabar todas las sesiones teóricas, editar y colgar las partes más importantes para que los alumnos no asistentes puedan seguirlas a posteriori.

Ciclos de Mejora en el Aula (2020). Experiencias de Innovación Docente de la US Esta obra se distribuye con la licencia Creative Commons 


\section{Sesión 4}

La sesión es muy positiva por la implicación de los alumnos en crear una sesión original y adecuada. Su principal fortaleza es la oportunidad que tienen los alumnos de mostrar su trabajo delante de sus compañeros.

La principal debilidad es la no aplicación de protocolos anticovid en las presentaciones y que solo se nombren las medidas de seguridad en las explicaciones de los ejercicios, pero con problemas para mantenerlas durante el desarrollo de los mismos. Una propuesta de mejora para posteriores cursos seria realizar una sesión de hierba y otra de sala.

\section{Evaluación del aprendizaje de los alumnos}

En las escaleras de aprendizaje se plasma la clasificación de las respuestas a las preguntas del cuestionario, se trata de reflejar un patrón común, desde los más simples a los más complejos, un grupo en cada escalón y se coloca a cada lado el porcentaje de estudiantes que están en ese nivel (se coloca una comparativa de porcentajes entre cuestionario inicial y final). Se analizan los obstáculos entre un nivel y otro y se formulan con una frase, formando la escalera completa. Nos ofrece una imagen muy gráfica y permite ver quien ha superado obstáculos, conociendo la evolución del estudiante.

En las escaleras de aprendizaje se muestran los ejemplos gráficos de las preguntas 4 y 5 del cuestionario, se observa la evaluación del aprendizaje comparando sus conocimientos iniciales y finales.

Ciclos de Mejora en el Aula (2020). Experiencias de Innovación Docente de la US Esta obra se distribuye con la licencia Creative Commons 
Pregunta 4. ¿Cómo enseñamos Hockey en nuestra escuela?

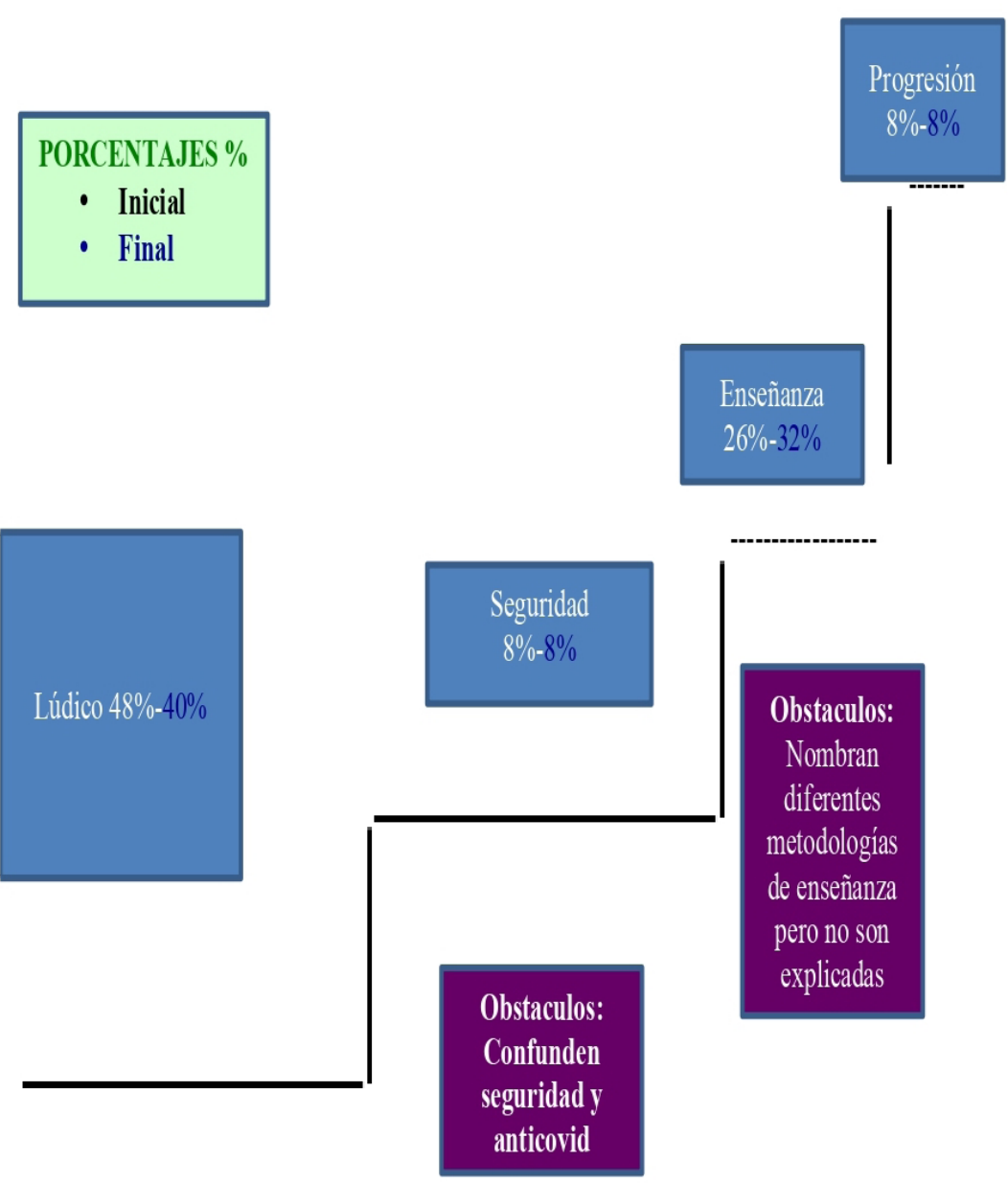

Figura 3. Escalera de aprendizaje y evaluación.

Análisis escalera: En esta pregunta, una de las principales de nuestro mapa de contenidos, se observa como los alumnos tienen claro sus modelos de aprendizaje aplicados a la enseñanza, partiendo desde el más común, de dar un aspecto lúdico a la actividad, progresando hacia tener en cuenta la seguridad, aunque confunden seguridad solo con medidas anticovid, pero en la práctica lo aplican de manera correcta, para superar este obstáculo y Ciclos de Mejora en el Aula (2020). Experiencias de Innovación Docente de la US
Esta obra se distribuye con la licencia Creative Commons Reconocimiento-NoComercial-SinObraDerivada Internacional (CC BY-NC-ND 4.0.) 
evolucionar hacia relacionar metodologías de enseñanza con la enseñanza del Hockey y, una vez superado, no saber explicar cual de ellas usar.

Pregunta 5. ¿Qué son para tilas técnicas de prevención y protocolo anticovid

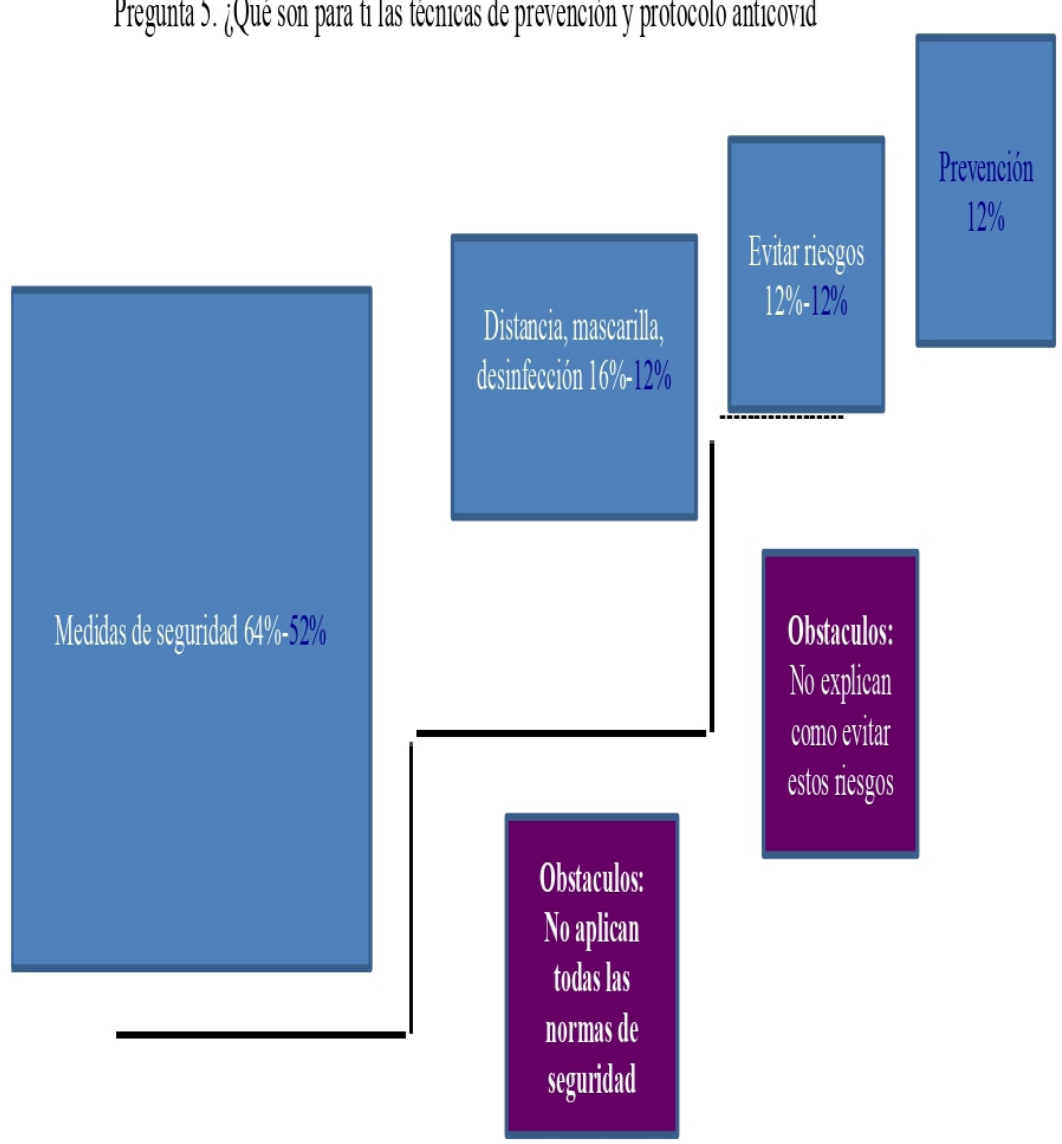

Figura 4. Escalera de aprendizaje y evaluación.

Análisis escalera: Otra pregunta muy importante en la época de pandemia y ante la necesidad de realizar prácticas experimentales presenciales es conocer las técnicas de prevención y protocolos anticovid. Todos debido conocen en la teoría la gran mayoría de medidas de seguridad (uso de mascarillas, desinfección, distancia, etc.), pero su principal obstáculo es que no las aplican en la práctica, solo las que se les obliga y se les recuerda a cada instante.

Ciclos de Mejora en el Aula (2020). Experiencias de Innovación Docente de la US Esta obra se distribuye con la licencia Creative Commons 
En el cuestionario final, algunos alumnos alcanzan el nivel referido a la necesidad de prevención y crean un propio protocolo anticovid aplicado a su presentación en la práctica. Siendo muy positiva y satisfactoria su evolución en esta pregunta

\section{Evaluación del CIMA}

\section{Cuestiones a mantener y cambios en futuros ciclos de mejora}

Para la posterior aplicación en cursos futuros, mantendría la realización de un diagnostico final e inicial, pero en mi caso ampliaría el periodo de tiempo entre ellos, y buscaría otros métodos de motivación para los alumnos, evaluación, etc. Ya que muchos lo han considerado un simple trámite o tarea repetitiva y se han limitado a repetir el mismo cuestionario inicial, desvirtuando la realidad bajo mi punto de vista, porque su desarrollo como alumno y su aprendizaje, como así han demostrado en la práctica, no se corresponde con lo expresado en el cuestionario.

Dar importancia a los modelos que favorecen la participación del alumno a través de la resolución de preguntas y el desarrollo de actividades tanto en la teoría como después llevarlo a la práctica para que experimenten y vivencien el aprendizaje, desarrollando la actividad.

La realización del diario de clase también aporta y enriquece el desarrollo de la clase por lo que lo mantendría y cambiaría las escaleras de evaluación y buscaría otro método, ya que me han resultado más dificultosas que útiles.

Seguiría con mi forma de evaluar, donde los trabajos que pido a los alumnos: tareas de búsqueda, dossier

Ciclos de Mejora en el Aula (2020). Experiencias de Innovación Docente de la US Esta obra se distribuye con la licencia Creative Commons 
de prácticas, video explicativo de un apartado técnico y presentación práctica, los evalúo con una rubrica que los alumnos conocen de antemano y saben que se les pide y cómo se evalúa, Y al final de la asignatura les paso a los alumnos un pequeño cuestionario anónimo, donde evalúen la asignatura, al docente y aporten sus propuestas de mejora para la asignatura.

Una tarea pendiente en la evaluación es introducir elementos de autoevaluación y coevaluación, pero cuando lo he intentado no ha sido fructífero y ha tenido resultados irreales. En futuros cursos lo añadiría, pero desde el anonimato, intentando aumentar la objetividad desde ese punto.

\section{Aspectos de la experiencia a incorporar en la práctica docente}

El uso de mapa de contenidos, la búsqueda de una mayor participación del alumnado en su aprendizaje, siendo paciente y dándole las bases o herramientas para que pueda llegar a sus propias opiniones y modelos mentales. Ser más visual y esquemático para llegar al alumno y plantearle retos para que el desarrolle el conocimiento

El uso de preguntas principales y relacionadas que sirvan para aumentar la interacción y participación del alumnado y que le clarifique aquello contenidos realmente interesantes e imprescindibles que es necesarios que adquieran para completar su aprendizaje a la vez que se les proporcionan herramientas para que profundicen en el conocimiento.

Ciclos de Mejora en el Aula (2020). Experiencias de Innovación Docente de la US Esta obra se distribuye con la licencia Creative Commons 


\section{Principios Didácticos}

Mis principios didácticos personales son los que han guiado la experiencia presente y permanecerán en el futuro tras ver lo positivo de los cambios introducidos, los principales son:

- Participación activa del alumno e implicación en la asignatura y en su aprendizaje

- Motivación e incentivación al alumno para que se implique en el desarrollo de la asignatura

- Uso de carpetas de aprendizaje/tareas de búsqueda donde se una el conocimiento, que sean trabajadas por el alumno

- Fomentar el trabajo en equipo, tanto en teoría como en la práctica

Ciclos de Mejora en el Aula (2020). Experiencias de Innovación Docente de la US Esta obra se distribuye con la licencia Creative Commons 
Palabras clave: Hockey, CAFD, Docencia Universitaria, Experimentación Docente, Innovación

Keywords: Hockey, CAFD, University Teaching, Teaching Experience, Innovation

\section{Referencias bibliográficas.}

Bain, K. (2006). Lo que hacen los mejores profesores universitarios. Valencia: Universidad de Valencia.

Finkel, D. (2008). Dar clase con la boca cerrada. Valencia: Universidad de Valencia.

Gallardo, L. (2020). ¿Qué son los Covid-free? Y que podemos hacer para la vuelta a la vida normal. Recuperado de: https://seed-deporte.es/que-son-los-covid-freey-que-podemos-hacer-para-la-vuelta-a-la-vida-nor$\mathrm{mal}$.

Gómez, J. L. (2020). Y de pronto el covid-19 nos hizo despertar. Revista Española de Educación Física y Deportes, 429, 95-100. Recuperado de: https://www.agesport.org/ y-de-pronto-el- covid-19-nos-hizo-despertar-por-joseuis-gomez-calvo/

Ministerio de Sanidad (2020). Procedimientos y medidas para la prevención y el control de la infección. Recuperado de: https://www.mscbs.gob.es/en/profesionales/ saludPublica/ccayes/alertasActual/nCov-China/documentos.htm.

Orden SND/380/2020, de 30 de abril, sobre las condiciones en las que se puede realizar actividad física no profesional al aire libre durante la situación de crisis sanitaria ocasionada por el COVID-19. Recuperado de: https:// boe.es/boe/dias/2020/05/01/pdfs/BOE-A-2020-4767. pdf

Porlán, R. (Coord), (2017). Enseñanza universitaria como mejorarla. Madrid: Ediciones Morata.

Real Federación Andaluza de Hockey (2020). Protocolo relativo a la protección y prevención de la salud frente al sars-cov-2 (covid-19) en el entrenamiento y/o competición de la Federación Andaluza de Hockey. Recuperado de: http://hockeyandalucia.es/wp-content/uploads/2020/09/PROTOCOLO-GENERAL-COVID-19.-FAH-2020.-VERSION-1.0-1.pdf

Ciclos de Mejora en el Aula (2020). Experiencias de Innovación Docente de la US Esta obra se distribuye con la licencia Creative Commons 